\title{
New Metrics for Meta-Analyses of Heterogeneous Effects
}

\author{
Maya B. Mathur ${ }^{1,2 *}$ and Tyler J. VanderWeele ${ }^{1}$
}

${ }^{1}$ Department of Epidemiology, Harvard T. H. Chan School of Public Health, Boston, MA, USA

${ }^{2}$ Quantitative Sciences Unit, Stanford University, Palo Alto, CA, USA

*: Corresponding author:

mmathur@stanford.edu

Quantitative Sciences Unit (c/o Inna Sayfer)

1070 Arastradero Road

Palo Alto, CA

94305

Keywords: heterogeneity, reporting, effect sizes 


\section{Abstract}

We provide two simple metrics that could be reported routinely in random-effects meta-analyses to convey evidence strength for scientifically meaningful effects under effect heterogeneity (i.e., a nonzero estimated variance of the true effect distribution). First, given a chosen threshold of meaningful effect size, meta-analyses could report the estimated proportion of true effect sizes above this threshold. Second, meta-analyses could estimate the proportion of effect sizes below a second, possibly symmetric, threshold in the opposite direction from the estimated mean. These metrics could help identify if: (1) there are few effects of scientifically meaningful size despite a "statistically significant" pooled point estimate; (2) there are some large effects despite an apparently null point estimate; or (3) strong effects in the direction opposite the pooled estimate regularly also occur (and thus, potential effect modifiers should be examined). These metrics should be presented with confidence intervals, which can be obtained analytically or, under weaker assumptions, using bias-corrected and accelerated (BCa) bootstrapping. Additionally, these metrics inform relative comparison of evidence strength across related meta-analyses. We illustrate with applied examples and provide an $\mathrm{R}$ package to compute the metrics and confidence intervals. 


\section{Introduction}

Random-effects meta-analyses aggregate evidence across studies measuring heterogeneous effects. Reporting usually focuses on the estimated mean of the distribution of true effects. However, under heterogeneity, others caution against exclusive focus on the estimated mean and recommend also reporting the estimated variance of true effects, not only the proportion of variance attributable to heterogeneity ${ }^{1,2}$. Summarizing evidence strength by comparing only the estimated mean to a threshold of scientific importance is needlessly dichotomous and has, in the past, led authors to conflicting conclusions in meta-analyses reporting nearly identical point estimates (see ${ }^{3,4}$ with additional commentary on dichotomization $\mathrm{by}^{5}$ ). Others have proposed informative summary metrics that consider heterogeneity by characterizing the range of effects in the distribution or providing a prediction interval for a new effect in the population or in a subgroup $^{1,2,6-8}$.

Extending these previous recommendations regarding heterogeneity in meta-analyses, we recommend also considering questions such as: "How common are effects of a size that is scientifically meaningful?" along with the traditional question: "What is the average effect size?" We therefore propose simple metrics that, unlike traditional metrics, directly address the former question. These metrics make inference to the population of true effect sizes while accounting for differences in precision across studies. As discussed below, this is distinct from simply "vote-counting" the significant $p$-values or the observed effect sizes (i.e., those measured with statistical error in the meta-analyzed studies) stronger than a threshold because the vote-counting approach, unlike our metrics, does not account for differences in precision and sample size across studies.

Reporting these new metrics along with traditional metrics may better identify situations in which (1) a "statistically significant" point estimate obscures the fact that the population of effect sizes contains few of scientifically meaningful size; or conversely (2) a null point estimate disguises the fact that, due to heterogeneity, there are large effect sizes in some settings. Additionally, these metrics help identify whether the treatment or exposure of interest may have scientifically meaningful effects in the opposite direction in some populations, pointing to the need to further examine potential effect modifiers. Lastly, they can inform comparison of relative evidence strength across related meta-analyses.

\section{Methods}

We make two recommendations to better characterize heterogeneous effect sizes represented by a given literature. First, in meta-analyses with heterogeneity (i.e., a nonzero estimate of $\tau$, the standard deviation of the true effects), the investigator could select a threshold above which an effect size might be considered of scientifically meaningful size and could report the estimated proportion of true effect sizes exceeding this threshold, along with a confidence interval. There is a large, interdisciplinary literature considering how to choose such thresholds, which we briefly summarize in the Online Supplement. For example, if the effect sizes are relative risks, then depending on the scientific context and 
informed by considerations such as those described in the Online Supplement, we might consider only those above 1.1 to be scientifically meaningful. Again depending on context, if fewer than (for example) $10 \%$ of true effect sizes surpass this threshold, we might consider evidence strength for scientifically meaningful effect sizes to be fairly weak.

Second, given a chosen threshold of scientific importance, we recommend that meta-analyses report the proportion of effect sizes stronger than a second (possibly symmetric) threshold on the opposite side of the null hypothesis (e.g. $1 / 1.1 \approx 0.9)$. That is, we might estimate a pooled relative risk $(R R)$ of 1.2 , indicating that the mean effect is positive. Yet with enough heterogeneity, the population of effects may also contain a nonneglible proportion of strong inverse associations (for example, $18 \%$ below a symmetric $R R$ of $1 / 1.1 \approx 0.9$ ). In practice, for both metrics, it may be informative to report results at more than one threshold of scientific importance as well as to report the meta-analytic estimates (particularly inference on the heterogeneity estimate) required to allow a reader to compute our proposed metrics for an arbitrary threshold.

These two proportions with their confidence intervals can be computed using only the estimated mean, heterogeneity, and their standard errors from a random-effects meta-analysis fit with any estimation approach that yields unbiased estimates of the mean and variance of the effect distribution. Specifically, under standard assumptions of parametric random-effects meta-analysis ${ }^{9}$, studies have true effect $\operatorname{sizes}^{1} \theta_{i}$ that are independently normal with a grand mean $\mu$ and variance $\tau^{2}$. Suppose we estimate $\mu$ with a point estimate denoted $\widehat{\mu}$ and estimate $\tau^{2}$ using one of several heterogeneity estimators ${ }^{10}$, denoted $\widehat{\tau}^{2}$. If $\widehat{\mu}$ is above the null value (e.g., an estimated $\log$ - $O R$ of $\log (1.3)$ ), then the first proposed metric, namely the estimated proportion of studies in the population with effect size greater than $q$ (e.g., $q=\log (1.1)$ ), is simply:

$$
\widehat{P}(\theta>q)=1-\Phi\left(\frac{q-\widehat{\mu}}{\sqrt{\widehat{\tau}^{2}}}\right), \widehat{\tau}^{2}>0
$$

where $\Phi$ denotes the standard normal cumulative distribution function. For the second metric, we can estimate the proportion below a second threshold $q^{*}$ as:

$$
\widehat{P}\left(\theta<q^{*}\right)=\Phi\left(\frac{q^{*}-\widehat{\mu}}{\sqrt{\widehat{\tau}^{2}}}\right), \widehat{\tau}^{2}>0
$$

In either case, asymptotic $95 \%$ confidence interval limits are: ${ }^{2}$

\footnotetext{
${ }^{1}$ Here, we reiterate the crucial distinction between the "true" effect sizes, which are unobservable statistical parameters, and the observed effect sizes measured with statistical error in each study. Variability in the true effect sizes comprises only heterogeneity, whereas variability in the observed effect sizes comprises both statistical error and heterogeneity. Thus, to estimate the proportion of true effects above or below a threshold, we must first use the observed effects to estimate the distribution of the true effects through the standard random-effects model.

${ }^{2}$ This standard error applies for estimators $\widehat{\tau}^{2}$ that are asymptotically normal and independent of $\widehat{\mu}$. This holds, for example, for the maximum likelihood estimators under the assumption that $E\left[\widehat{\theta}_{i} \mid \sigma_{i}^{2}\right]=E\left[\widehat{\theta}_{i}\right]$, where $\widehat{\theta}_{i}$ and $\sigma_{i}^{2}$ respectively denote the point estimate and squared standard error of the $i^{t h}$ study (Online Supplement). In practice, this assumption can be verified by inspecting a funnel
} 


$$
\widehat{P} \pm 1.96 \sqrt{\frac{\widehat{\operatorname{Var}}(\widehat{\mu})}{\widehat{\tau}^{2}}+\frac{\widehat{\operatorname{Var}}\left(\widehat{\tau}^{2}\right)(\widehat{\mu}-q)^{2}}{4\left(\widehat{\tau}^{2}\right)^{3}}} \cdot \phi\left(\frac{q-\widehat{\mu}}{\sqrt{\widehat{\tau}^{2}}}\right)
$$

where $\phi$ denotes the standard normal density function. $\widehat{P}$ in this expression can be computed from either Equation (1) or Equation (2), and $q$ is replaced by $q^{*}$ when considering the second metric. Simulation results (Online Supplement) indicate that when the number of studies is less than $10, \widehat{P}<0.15$, or $\widehat{P}>0.85$, it is preferable to estimate the confidence interval via bias-corrected, accelerated bootstrapping ${ }^{11,12}$. Applied examples in this paper for which $\widehat{P}<0.15$ or $\widehat{P}>0.85$ use bootstrapped confidence intervals, for which code is available online (https://osf.io/674gt/).

If $\widehat{\mu}$ is below rather than above the null value (for example, we estimate a mean $\log -O R$ of $\log (0.85)$ ), then we would simply switch the two equations, using Equation (2) to estimate the proportion of effects below a threshold (e.g., a $\log -O R$ of $\log (0.90)$ ) and Equation (1) to estimate the proportion of effects above a second threshold. (As usual, we use the log scale when considering odds ratios for approximate normality.) In practice, these proportions are easy to compute manually or using the $\mathrm{R}$ function prop_stronger, available in an open-source public repository (https://osf.io/pr2s9/). We now illustrate how they can facilitate interpretation through three examples.

\section{Example 1: A "significant" mean despite little evidence for strong effects}

Meta-analyses often achieve large pooled sample sizes and high power, and thus it can happen that a very small estimated mean attains "statistical significance" at a given significance threshold. The proposed metrics may then illustrate that despite a "significant" $p$-value, few effects are in fact strong enough to warrant scientific interest. For example, a recent meta-analysis ${ }^{13}$ estimated a mean correlation of $r=-0.06(p=0.01)$ between increased psychological stress and shorter telomeres, and subsequent literature largely interpreted this finding as supportive of an association. However, using the proposed methods, we can estimate that only $6 \%$ (95\% CI: $0 \%, 71 \%$ ) of true correlations between stress and telomere length are stronger than the modest threshold of $r=-0.10$, and that almost none $(0 \%, 95 \%$ CI: $0 \%, 46 \%$ ) are stronger than $r=-0.20$. It is important to note that the upper confidence interval limits for both choices of threshold represent non-negligible proportions, so these results should not be interpreted as evidence against the occurrence of correlations stronger than these thresholds. The $95 \%$ prediction interval is $(-0.13,0.02)$; this additionally suggests that, with high probability, a new effect drawn from the population will be near the null ${ }^{6,7}$. These metrics perhaps better qualify the scientific relevance of the meta-analyzed studies. Note that comparing the point estimate alone to the threshold would not adequately convey evidence strength: this meta-analysis estimated little heterogeneity ( $\tau=0.03)$ and hence the small point estimate suggests a small proportion of strong true effects, but if the heterogeneity estimate had been larger (for example, three-fold larger on the $\tau$ scale), then the estimated percent of true correlations definition of $\sigma_{i}^{2}$.) When these assumptions are violated, the BCa confidence interval should be used instead. 
stronger than $r=-0.10$ would increase from a negligible $6 \%$ to $30 \%$ (95\% CI: $13 \%, 46 \%){ }^{3}$

\section{Example 2: Meta-analyses with different conclusions despite similar ev- idence strength}

These metrics can also inform comparison across meta-analyses, which is of interest when a meta-analysis is updated to reflect new literature, when applying different inclusion criteria or analysis methods to the same literature, or when meta-analyses assess different, but related, outcomes. A common, but unsatisfactory, approach is to compare only estimated means and "statistical significance". However, around the $p$-value cut-off (e.g., 0.05), "statistical significance" can be highly sensitive to the inclusion of even one additional small study. Moreover, these metrics are not robust to the influence of large or outlying studies; they may therefore suggest a spuriously large discrepancy between metaanalyses. Yet even when estimated means differ, the more stable proposed metrics may be similar and have substantially overlapping confidence intervals, suggesting less dramatic discrepancies. Alternatively, despite comparable point estimates, differences in heterogeneity across meta-analyses can lead to different proportions of strong effect sizes.

We illustrate using two meta-analyses investigating the effect of omega-3 fatty acid supplementation on depression in randomized trials; the first meta-analysis ${ }^{14}$ suggested a "significant" beneficial effect (standardized mean difference $(S M D)=0.26,95 \%$ CI: 0.12, 0.39), whereas the second ${ }^{15} \operatorname{did} \operatorname{not}(S M D=0.25,95 \% \mathrm{CI}:-0.07,0.56)$. Using $S M D=0.50$ as a threshold of scientific importance (based, for instance, on a minimum subjectively perceptible difference; see Online Supplement), our first metric estimates that 22\% (95\% CI: 6\%, 38\%) of $S M D$ s in the former meta-analysis ${ }^{14}$ surpass this threshold, comparable to $30 \%(95 \%$ CI: $4 \%, 56 \%)$ in the latter ${ }^{15}$ (Figure 1). Using our second metric, neither meta-analysis suggests a high proportion of strong detrimental effects (1\% below $S M D=-0.50$ with $95 \%$ CI: $0 \%, 3 \%$ in the former and $6 \%$ with 95\% CI: $0 \%, 22 \%$ in the latter). Thus, evidence strength in these two meta-analyses is perhaps more concordant than their disagreement on "statistical significance" alone suggests. (A supplementary example regarding comparison between meta-analyses with differing point estimates appears in the Online Supplement.)

\footnotetext{
${ }^{3}$ It may appear surprising that the latter confidence interval under increased heterogeneity is narrower than the confidence interval with the observed estimates. This occurs for two reasons. First, $\widehat{\operatorname{Var}}\left(\widehat{\tau}^{2}\right)$ is not estimable for the hypothetical example with increased heterogeneity, so we held it constant to its observed value, resulting in a relatively small $\widehat{\operatorname{Var}}\left(\widehat{\tau}^{2}\right)$ compared to $\widehat{\tau}^{2}$. Second, an estimated true effect distribution with increased heterogeneity has flatter tails, resulting in more stable estimates of $\widehat{P}\left(\theta<q^{*}\right)$ or $\widehat{P}(\theta>q)$ in some neighborhoods of $\widehat{\mu}$.
} 
Figure 1: Estimated proportion of standardized mean differences (shaded) stronger than threshold of scientific importance at $S M D=0.50$ (solid red line) in two meta-analyses with differing "statistical significance". Dashed red line: reference null value $(S M D=0)$.

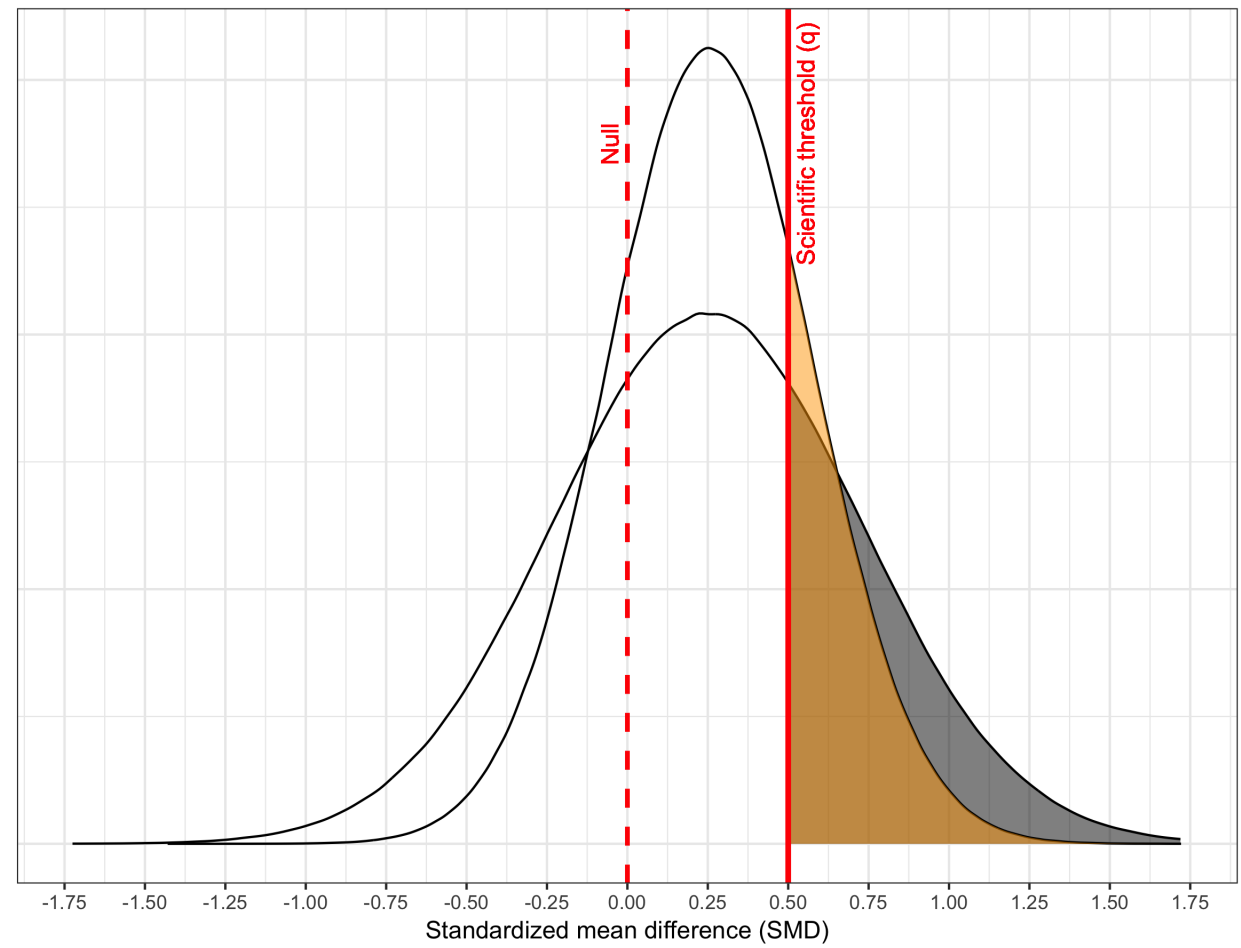

\section{Example 3: A wide prediction interval despite evidence for many strong effects}

Others recommend reporting a prediction interval representing a plausible range for a new effect drawn from the distribution underlying the meta-analyzed studies ${ }^{2,6,7}$. We agree, while also noting that our metrics convey additional information. For example, a meta-analysis ${ }^{16}$ of 19 trials on the effect of intravenous magnesium on mortality following acute myocardial infarction estimated an odds ratio of 0.72 (95\% CI: 0.58, 0.90), corresponding to a $95 \%$ prediction interval of $(0.42,1.25)$. This interval is fairly wide and substantially overlaps the null, indicating considerable uncertainty about the size and direction of an effect in a new study. Nevertheless, if we consider inverse associations below $O R=0.8$ to be scientifically meaningful, our proposed metrics suggest that a high proportion of true effects ( $66 \%$ with $95 \%$ CI: $28 \%$, $100 \%)$ are more protective than this threshold and that few are comparably strong in the opposite direction ( $2 \%$ above $O R=1.2,95 \% \mathrm{CI}: 0 \%, 7 \%)$. These metrics suggest that there is strong evidence that a substantial proportion of studies have reasonably large protective effects, even though the prediction interval for any single true effect includes the null value of $O R=1$. We provide this example for illustrative purposes only, noting that others have raised methodological concerns about this literature, including, for example, publication bias and possible data quality issues in some included studies ${ }^{17}$. 
The findings of the prediction interval and of our metrics are not contradictory, but complementary: the prediction interval infers plausible values for a single effect by considering the middle $95 \%$ of the area of the effect distribution, whereas our metrics estimate the area of the lower and upper tails. Intuitively, as the effect distribution becomes more heterogeneous, the middle 95\% widens, yielding a wider prediction interval. Simultaneously, the tails thicken, increasing the proportion of strong effects in both the same and the opposite direction as the estimated mean. Thus, with enough heterogeneity, an estimated mean near the null can belie the existence of meaningful effect sizes in some settings. In such cases, the first and second proposed metrics would likely indicate a substantial proportion of strong effect sizes both in the same and in the opposite direction from the estimated mean; this pattern of results would invite exploration of reasons for heterogeneity, for example through individual patient data meta-analysis with covariates or meta-regression.

\section{Technical points}

The proposed metrics are distinct from antiquated "vote-counting" procedures based on the proportion of studies with significant $p$-values. Such methods fail to differentiate small from large effect sizes (counting only those that are "statistically significant") and fail to account for differences in precision and sample size across studies ${ }^{1}$. A modified procedure could consider the proportion of observed effect sizes (rather than $p$-values) above a threshold of scientific interest, but this would still limit attention to the observed effect sizes despite that these are measured with sampling error. In contrast, the proposed metrics make appropriately weighted inferences regarding the distribution of true effect sizes in the population (rather than of the observed estimates). In the telomere length example, a basic vote count finds that $14 \%$ of studies ( 3 of 21 ) had $p<0.05$. A count of observed effect sizes stronger than a threshold, but ignoring sample sizes and sampling variability, finds that $24 \%$ ( 5 of 21 ) are below $r=-0.20$. In contrast, our approach concerns the true effect sizes and estimates that $<0.1 \%$ of these are stronger than ${ }^{4} r=-0.20$.

These metrics are model-based; they assume that the true effect sizes are normally distributed. In practice, this implies the sometimes testable assumption that the observed effect sizes are normal. Because this distributional assumption may be inexact, our proposed metrics are perhaps most usefully treated as summary metrics of evidence strength for effects of scientifically meaningful size rather than as precise proportions. Additionally, these metrics and inference require the meta-analytic model to be statistically valid, but estimating the heterogeneity without bias, in particular, can be challenging ${ }^{10}$. It is thus important to choose a heterogeneity estimator with good statistical properties for the chosen outcome type ${ }^{10}$ to enable good performance of our metrics as well as of traditional metrics. Last, publication bias can compromise our metrics in the same way that it compromises standard analyses. Therefore, when publication bias is suspected, we recommend conducting sensitivity analyses that estimate $\widehat{\mu}$ and $\widehat{\tau}^{2}$ in a manner that corrects for publication bias (e.g., ${ }^{18}$ ). These bias-corrected estimates can then be used to unbiasedly estimate our proposed metrics.

\footnotetext{
${ }^{4}$ Due to the theoretical connection between random-effects meta-analysis and mixed models, this discrepancy can also be viewed as shrinkage of the random effect estimates toward $\widehat{\mu}$. Indeed, in the telomere length meta-analysis, the best linear unbiased predictions of the study effect sizes were on average $71 \%$ as large absolutely as the corresponding point estimates.
} 


\section{Conclusion}

To better characterize heterogeneous effects in meta-analysis, we recommend supplementing standard reporting with two simple metrics regarding the proportion of effects above a threshold of scientific importance and below a second threshold on the opposite side of the null. These metrics account for effect size, heterogeneity, and statistical error, and they are easy to compute manually or or using the R function prop_stronger.

\section{Acknowledgements}

We are grateful to Dr. Ian Shrier for insightful comments that significantly improved the narrative.

\section{Reproducibility}

All data and code required to reproduce the applied examples are publicly available (https://osf.io/ksyq5/), as is full documentation for the telomere dataset (https://osf.io/6937j/).

\section{Funding}

MM was supported by National Defense Science and Engineering Graduate Fellowship 32 CFR 168a. TVW was supported by NIH grant ES017876 and CA222147. The funders had no role in the design, conduct, or reporting of this research. 


\section{References}

1. Borenstein M, Hedges L, Higgins J, Rothstein H. Introduction to Meta-Analysis. Wiley Online Library; 2009.

2. Higgins J, Thompson S, Spiegelhalter D. A re-evaluation of random-effects meta-analysis. Journal of the Royal Statistical Society: Series A (Statistics in Society). 2009;172(1):137-159.

3. Kirsch I, Deacon B, Huedo-Medina T, Scoboria A, Moore T, Johnson B. Initial severity and antidepressant benefits: A meta-analysis of data submitted to the Food and Drug Administration. PLoS Medicine. 2008;5(2):e45.

4. Turner E, Matthews A, Linardatos E, Tell R, Rosenthal R. Selective publication of antidepressant trials and its influence on apparent efficacy. New England Journal of Medicine. 2008;358(3):252-260.

5. Turner R, Rosenthal R. Efficacy of antidepressants. BMJ. 2008;336(7643):516.

6. Riley R, Higgins J, Deeks J. Interpretation of random effects meta-analyses. BMJ. 2011;342:d549.

7. IntHout J, Ioannidis J, Rovers M, Goeman J. Plea for routinely presenting prediction intervals in meta-analysis. BMJ Open. 2016;6(7):e010247.

8. Ades A, Lu G, Higgins J. The interpretation of random-effects meta-analysis in decision models. Medical DecisionMaking. 2005;25(6):646-654.

9. Sutton AJ, Abrams KR, Jones DR, Jones DR, Sheldon TA, Song F. Methods for meta-analysis in medical research. 2000.

10. Veroniki A, Jackson D, Viechtbauer W, et al. Methods to estimate the between-study variance and its uncertainty in meta-analysis. Research Synthesis Methods. 2016;7(1):55-79.

11. Efron B. Better bootstrap confidence intervals. Journal of the American Statistical Association. 1987;82(397):171-185.

12. Carpenter J, Bithell J. Bootstrap confidence intervals: When, which, what? A practical guide for medical statisticians. Statistics in Medicine. 2000;19(9):1141-1164.

13. Mathur M, Epel E, Kind S, et al. Perceived stress and telomere length: A systematic review, meta-analysis, and methodologic considerations for advancing the field. Brain, Behavior, and Immunity. 2016;54:158-169.

14. Appleton K, Rogers P, Ness A. Updated systematic review and meta-analysis of the effects of n-3 long-chain polyunsaturated fatty acids on depressed mood. The American Journal of Clinical Nutrition. 2010:ajcn-28313.

15. Bloch M, Hannestad J. Omega-3 fatty acids for the treatment of depression: Systematic review and meta-analysis. Molecular Psychiatry. 2012;17(12):1272.

16. Shrier I, Boivin J, Platt R, et al. The interpretation of systematic reviews with meta-analyses: An objective or subjective process? BMC Medical Informatics and Decision-Making. 2008;8(1):19.

17. Higgins JP, Spiegelhalter DJ. Being sceptical about meta-analyses: A Bayesian perspective on magnesium trials in 
myocardial infarction. International Journal of Epidemiology. 2002;31(1):96-104.

18. Vevea JL, Hedges LV. A general linear model for estimating effect size in the presence of publication bias. Psychometrika. 1995;60(3):419-435. 


\title{
Online Supplement for: "New Metrics for Meta-Analyses of Heterogeneous Effects"
}

\author{
Maya B. Mathur \& Tyler J. VanderWeele
}

\section{Contents}

Derivation of confidence interval

Forest plots for applied examples

Supplementary example: Meta-analyses with different estimated means, but similar evidence strength

Simulation study

References 


\section{Derivation of confidence interval}

In Lemma 1, we first establish conditions under which $\widehat{\mu}$ and $\widehat{\tau}^{2}$ are asymptotically independent.

Lemma 1. Let $\widehat{\mu}$ and $\widehat{\tau}^{2}$ denote maximum likelihood estimates under a normal specification in which, as usual, the within-study variances $\sigma_{i}^{2}$ are considered fixed and known (e.g., ${ }^{1}$ ). Suppose there are $k$ studies, with $\widehat{\theta}_{i}$ denoting the point estimate of the $i^{t h}$ study, and assume that $E\left[\widehat{\theta}_{i} \mid \sigma_{i}^{2}\right]=E\left[\widehat{\theta}_{i}\right]$. Then $\widehat{\mu}$ and $\widehat{\tau}^{2}$ are asymptotically independent.

Proof. The joint log-likelihood and partial derivatives are:

$$
\begin{aligned}
\log \mathcal{L}\left(\mu, \tau^{2}\right) & =-\frac{1}{2} \sum_{i=1}^{k} \log \left(2 \pi\left(\sigma_{i}^{2}+\tau^{2}\right)\right)-\frac{1}{2} \sum_{i=1}^{k} \frac{\left(\widehat{\theta}_{i}-\mu\right)^{2}}{\sigma_{i}^{2}+\tau^{2}} \\
\frac{\partial \log \mathcal{L}}{\partial \mu} & =-\frac{1}{2} \sum_{i=1}^{k}\left(\sigma_{i}^{2}+\tau^{2}\right)^{-1}\left(-2 \widehat{\theta}_{i}+2 \mu\right) \\
\frac{\partial^{2} \log \mathcal{L}}{\partial \mu \partial \tau^{2}} & =\frac{1}{2} \sum_{i=1}^{k}\left(\sigma_{i}^{2}+\tau^{2}\right)^{-2}\left(-2 \widehat{\theta}_{i}+2 \mu\right) \\
& =-\frac{1}{2} \sum_{i=1}^{k} \frac{2 \widehat{\theta}_{i}-2 \mu}{\sigma_{i}^{4}+2 \sigma_{i}^{2} \tau^{2}+\tau^{4}}
\end{aligned}
$$

The off-diagonal element of the expected Fisher information matrix is therefore:

$$
\begin{aligned}
\mathcal{I}_{12} & =-E\left[\frac{\partial^{2} \log \mathcal{L}}{\partial \mu \partial \tau^{2}}\right] \\
& =\frac{1}{2} k E\left[\frac{2 \widehat{\theta}_{i}-2 \mu}{\sigma_{i}^{4}+2 \sigma_{i}^{2} \tau^{2}+\tau^{4}}\right]
\end{aligned}
$$

By a second-order Taylor series expansion, we have, for general random variables $X$ and $Y$ :

$$
E[X / Y] \approx \frac{E[X]}{E[Y]}-\frac{\operatorname{Cov}(X, Y)}{E[Y]^{2}}+\frac{\operatorname{Var}(Y) E[X]}{E[Y]^{3}}
$$


We have $\mathrm{E}\left[2 \widehat{\theta}_{i}-2 \mu\right]=0$, so applying Equation (1) with the first and third terms equal to 0 yields:

$$
\begin{aligned}
\mathcal{I}_{12} & \approx \frac{1}{2} k \frac{E\left[\left(2 \mu-2 \widehat{\theta}_{i}\right)\left(\sigma_{i}^{4}+2 \sigma_{i}^{2} \tau^{2}+\tau^{4}\right)\right]}{E\left[\sigma_{i}^{4}+2 \sigma_{i}^{2} \tau^{2}+\tau^{4}\right]^{2}} \\
& =\frac{1}{2} k \frac{2 \mu E\left[\sigma_{i}^{4}\right]+4 \mu \tau^{2} E\left[\sigma_{i}^{2}\right]+2 \mu \tau^{4}-2 \tau^{4} E\left[\widehat{\theta}_{i}\right]-4 \tau^{2} E\left[\widehat{\theta}_{i} \sigma_{i}^{2}\right]-2 E\left[\widehat{\theta}_{i} \sigma_{i}^{4}\right]}{E\left[\sigma_{i}^{4}+2 \sigma_{i}^{2} \tau^{2}+\tau^{4}\right]^{2}} \\
& =\frac{1}{2} k \frac{2 \mu E\left[\sigma_{i}^{4}\right]+4 \mu \tau^{2} E\left[\sigma_{i}^{2}\right]+2 \mu \tau^{4}-2 \tau^{4} \mu-4 \tau^{2} \mu E\left[\sigma_{i}^{2}\right]-2 \mu E\left[\sigma_{i}^{4}\right]}{E\left[\sigma_{i}^{4}+2 \sigma_{i}^{2} \tau^{2}+\tau^{4}\right]^{2}} \\
& =0
\end{aligned}
$$

The penultimate line follows from the assumption that $E\left[\widehat{\theta}_{i} \mid \sigma_{i}^{2}\right]=E\left[\widehat{\theta}_{i}\right]$. Since the maximum likelihood estimates are asymptotically bivariate normal, asymptotic independence is established.

We now derive an asymptotic confidence interval for $\widehat{P}\left(\theta<q^{*}\right)$, which is identical to that of $\widehat{P}(\theta>q)$ for a symmetric choice of $q$. We assume use of the standard Dersimonian-Laird estimator, $\widehat{\mu}$, and an arbitrary estimator $\widehat{\tau}^{2}$ such that, asymptotically:

$$
\left[\begin{array}{c}
\widehat{\mu}-\mu \\
\widehat{\tau}^{2}-\tau^{2}
\end{array}\right] \approx N(\left[\begin{array}{l}
0 \\
0
\end{array}\right], \underbrace{\left[\begin{array}{cc}
\operatorname{Var}(\widehat{\mu}) & \operatorname{Cov}\left(\widehat{\mu}, \widehat{\tau}^{2}\right) \\
\operatorname{Cov}\left(\widehat{\mu}, \widehat{\tau}^{2}\right) & \operatorname{Var}\left(\widehat{\tau}^{2}\right)
\end{array}\right]}_{\Sigma / k})
$$

(Asymptotic normality is theoretically justified for the maximum likelihood and restricted maximum likelihood estimators $\widehat{\tau}^{2}$ and, in simulations, also appears to hold under the same conditions for the estimators proposed by references 2, 3, 4, and 5.) Apply the delta method: 


$$
\begin{aligned}
h\left(x_{1}, x_{2}\right) & =\widehat{P}\left(\theta<q^{*}\right) \\
& =\Phi\left(\frac{q^{*}-x_{1}}{\sqrt{x_{2}}}\right) \\
\nabla=\left[\begin{array}{c}
\frac{\partial h}{\partial x_{1}} \\
\frac{\partial h}{\partial x_{2}}
\end{array}\right] & =\left[\begin{array}{c}
-x_{2}^{-1 / 2} \phi\left(\frac{q^{*}-x_{1}}{\sqrt{x_{2}}}\right) \\
\frac{x_{1}-q^{*}}{2} x_{2}^{-3 / 2} \phi\left(\frac{q^{*}-x_{1}}{\sqrt{x_{2}}}\right)
\end{array}\right] \\
\sqrt{k}\left[h\left(\widehat{\mu}, \widehat{\tau}^{2}\right)-h\left(\mu, \tau^{2}\right)\right] & \rightarrow N\left(0,\left.\nabla^{\prime} \Sigma \nabla\right|_{\mu, \tau^{2}}\right) \\
\nabla^{\prime} \Sigma \nabla & =\nabla_{1}\left(\nabla_{1} \Sigma_{11}+\nabla_{2} \Sigma_{21}\right)+\nabla_{2}\left(\nabla_{1} \Sigma_{12}+\nabla_{2} \Sigma_{22}\right) \\
& =\nabla_{1}^{2} \Sigma_{11}+\nabla_{2}^{2} \Sigma_{22}
\end{aligned}
$$

for choices of estimators $\widehat{\tau}^{2}$ that are asymptotically independent of $\widehat{\mu}$. Thus:

$$
\widehat{\operatorname{Var}}\left(\widehat{P}\left(\theta<q^{*}\right)\right) \approx\left(\phi\left(\frac{q^{*}-\widehat{\mu}}{\sqrt{\widehat{\tau}^{2}}}\right)\right)^{2} \cdot\left(\frac{\widehat{\operatorname{Var}}(\widehat{\mu})}{\widehat{\tau}^{2}}+\frac{\widehat{\operatorname{Var}}\left(\widehat{\tau^{2}}\right)\left(\widehat{\mu}-q^{*}\right)^{2}}{4\left(\widehat{\tau}^{2}\right)^{3}}\right)
$$

Thus, approximate $95 \%$ confidence limits for $\widehat{P}\left(\theta<q^{*}\right)$ are:

$$
\widehat{P}\left(\theta<q^{*}\right) \pm \Phi^{-1}(0.975) \cdot \phi\left(\frac{q^{*}-\widehat{\mu}}{\sqrt{\widehat{\tau}^{2}}}\right) \cdot \sqrt{\frac{\widehat{\operatorname{Var}}(\widehat{\mu})}{\widehat{\tau}^{2}}+\frac{\widehat{\operatorname{Var}}\left(\widehat{\tau^{2}}\right)\left(\widehat{\mu}-q^{*}\right)^{2}}{4\left(\widehat{\tau}^{2}\right)^{3}}}
$$

An analogous derivation or argument from symmetry yields the same standard error for $\widehat{P}(\theta>q)$.

\section{Methods for choosing an effect size threshold}

Much existing work, spanning a variety of disciplinary perspectives, has discussed how to choose thresholds for scientifically meaningful effect sizes. Reference 6 provides an excellent review and examples of numerous methods in the context of health outcomes. In particular, they discuss a variety of "anchoring-based" methods in which an effect size threshold is chosen by relating the outcome measure to external criteria bearing immediate scientific or clinical relevance. For example, a minimum effect size threshold for a composite scale outcome could be defined in relation to naturally occuring discrepancies in the outcome between patient groups with and without a diagnosis ${ }^{7}$ or to effect sizes produced by existing interventions similar to the intervention under investigation ${ }^{8}$. In the telomere length example discussed in the main text, we 
might define a threshold of scientific importance through comparison to the correlation strength of age with telomere length (approximately $r=-0.34$ to -0.38$)^{9}$ since age is perhaps the best-established determinant of telomere degradation. We might expect a psychological state, such as stress, to have a somewhat weaker effect size than the biological process of aging. Thus, to select an effect size threshold for psychological stress, we might reduce the magnitude of the age-telomere length correlation to, for example, $r=-0.10$ or $r=-0.20$. Numerous other types of external "anchoring" criteria have also been used in the medical literature ${ }^{6}$.

When the population public health impact of a health condition is the primary concern, investigators could draw upon the extensive literature on cost-effectiveness decision rules in selecting an effect size threshold. For example, much existing work has discussed or empirically quantified the cost threshold at which societies (or individuals) are willing to pay for a specific improvement in health, such as an addition of one quality-adjusted life-year (e.g., ${ }^{10,11}$ ). Such findings could be used to "convert" hypothetical statistical effect sizes for a given health outcome to a concrete financial scale, such as dollars. A minimum effect size threshold could then be defined in relation to the utility, expressed in dollars, of the intervention or exposure of interest.

In contrast, in other scientific contexts, individuals' subjective experience of pain, distress, or disability may be the primary concern rather than (or in addition to) aggregate public health impact. In these cases, it may be useful to set the threshold as the minimum effect size that is subjectively perceptible ${ }^{12-14}$. A systematic review considered 62 studies that attempted to estimate such thresholds for a wide variety of health outcomes, for example by relating patients' subjective self-assessments to objective measurements of health condition severity ${ }^{14}$. This review found that $S M D=0.50$ was a surprisingly consistent minimally detectable effect size for health outcomes, perhaps reflecting fundamental mechanisms of human sensory discrimination or constraints on categorical discrimination due to working memory capacity. For ease of comparison to other statistical measures of effect size, the threshold $S M D=0.50$ is approximately equivalent (under some distributional assumptions) $)^{15,16}$ to an odds ratio of 2.5 or to a risk ratio of 1.6. However, it is important to note that an intervention that has only small effects on the individual level, even ones that are not subjectively perceptible, may still have very substantial impacts on a population public health level; thus, as described above, much lower thresholds might often be considered.

\section{Forest plots for applied examples}

Below are forest plots corresponding to each applied example presented in the main text and Supplement. 
Supplementary Figure 1: Perceived stress and telomere length (Example 1)

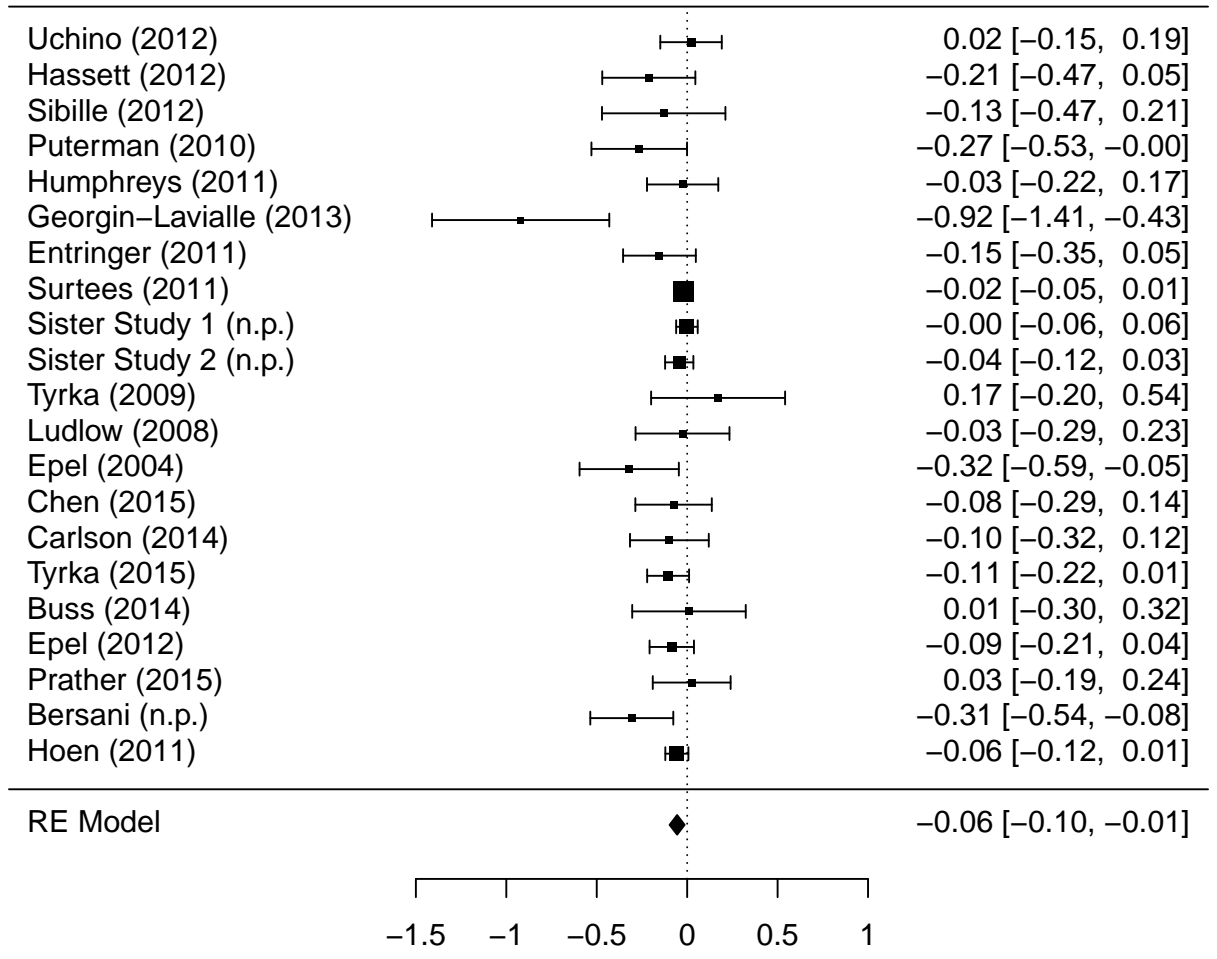

Fisher's z Transformed Correlation Coefficient 
Supplementary Figure 2: Omega-3 supplementation and depression (Example 2, first meta-analysis)

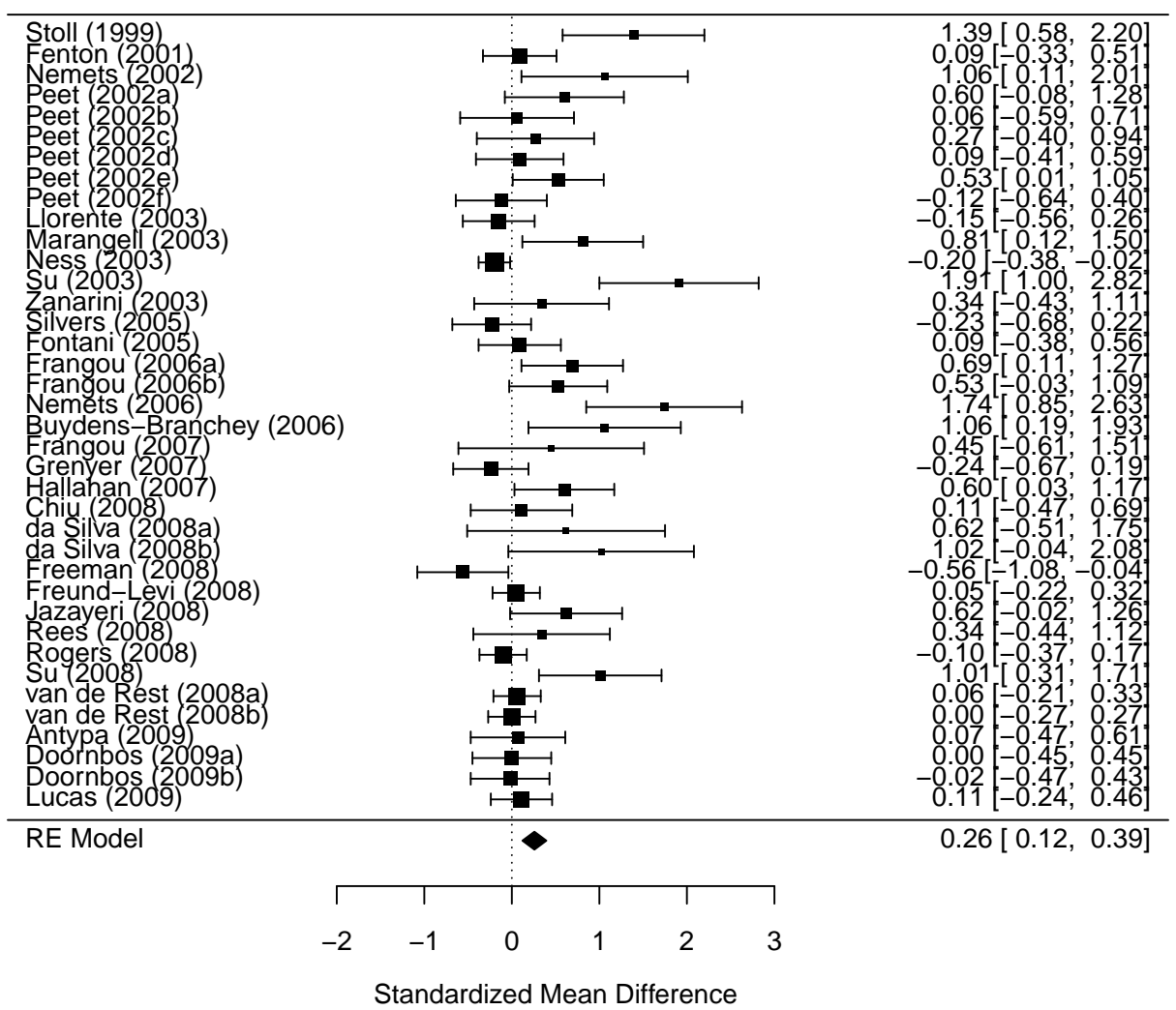


Supplementary Figure 3: Omega-3 supplementation and depression (Example 2, second meta-analysis)

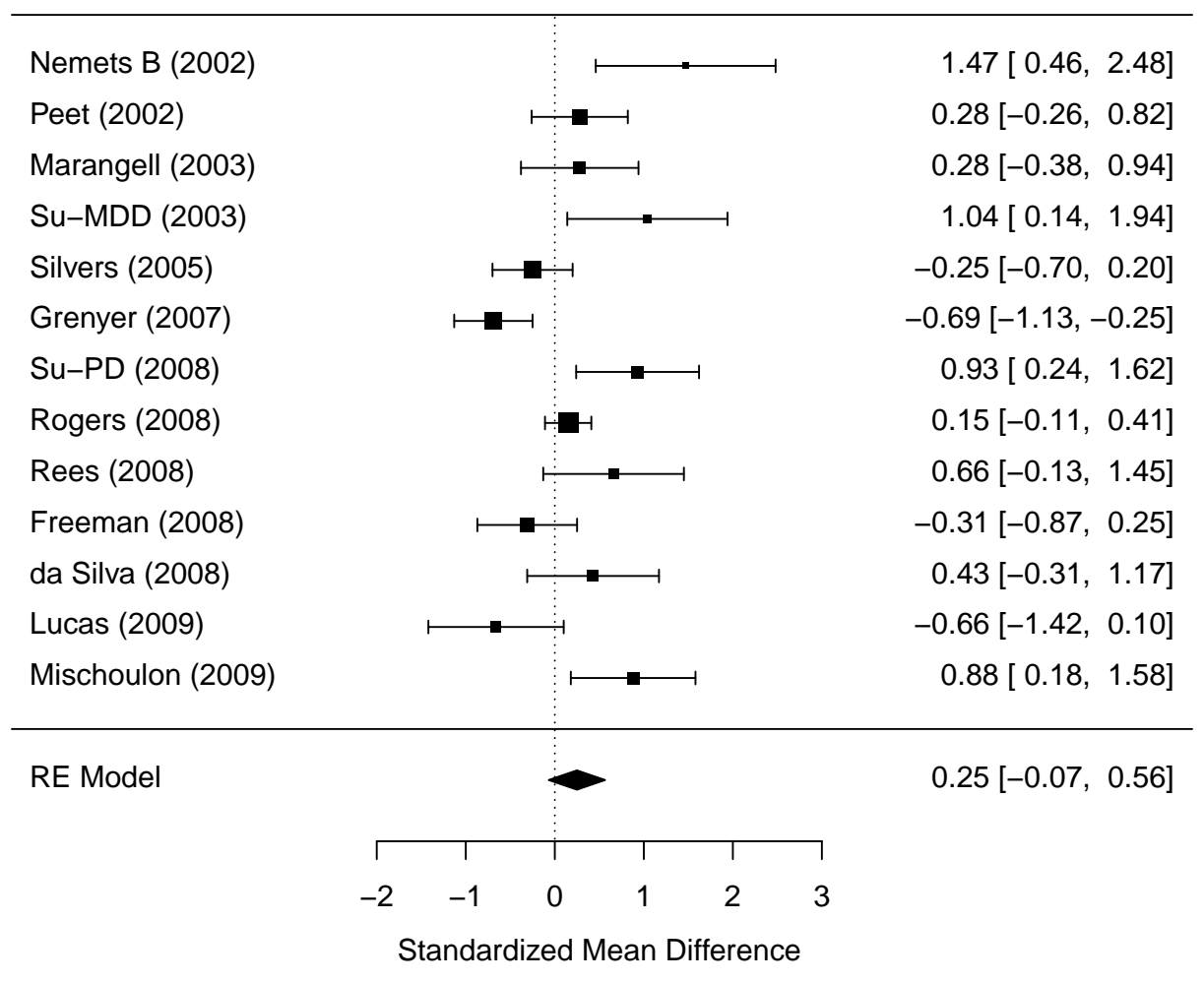


Supplementary Figure 4: Magnesium and myocardial infarction (Example 3, 19-study meta-analysis)

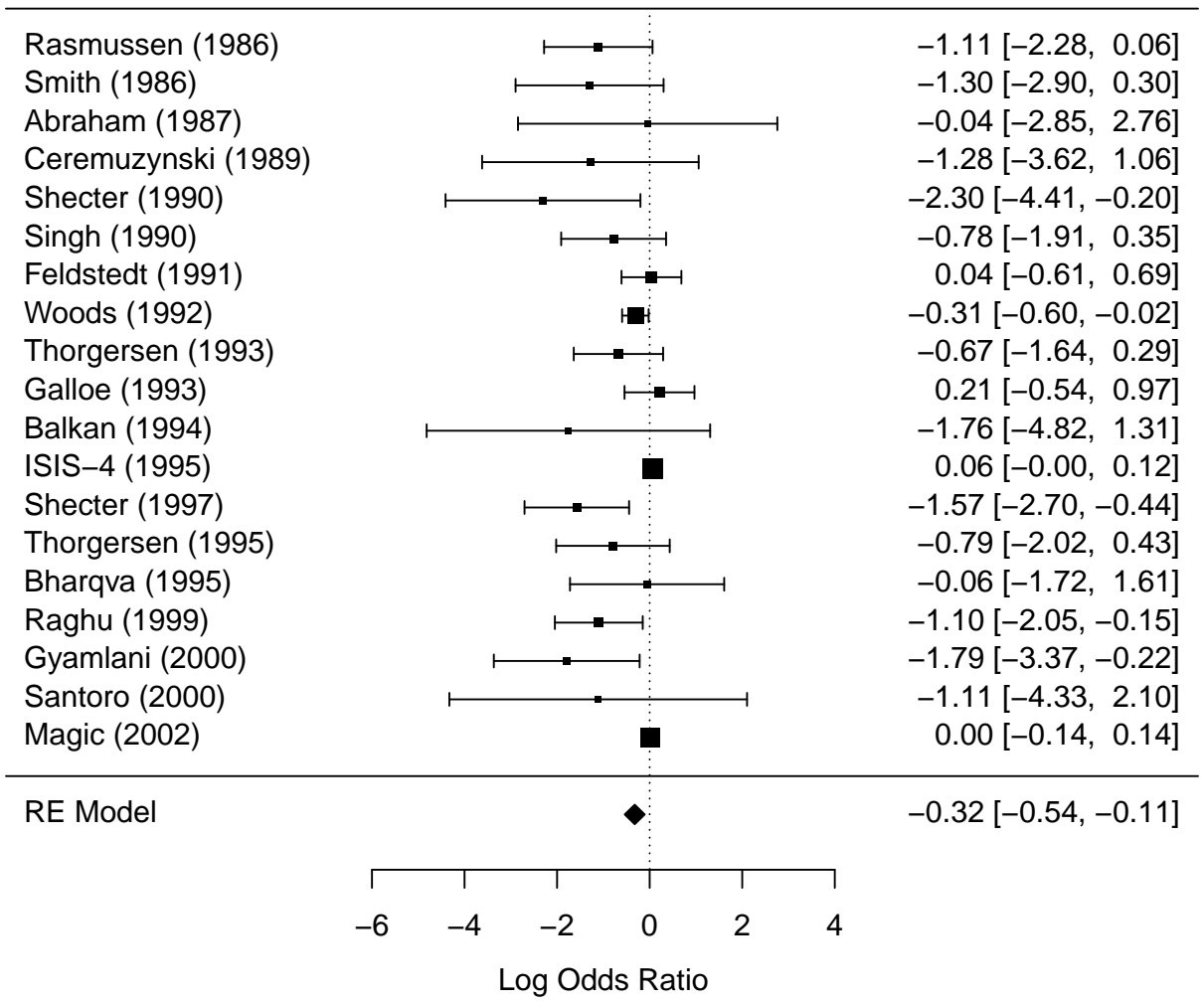


Supplementary Figure 5: Magnesium and myocardial infarction (Supplementary Example, 16-study metaanalysis)

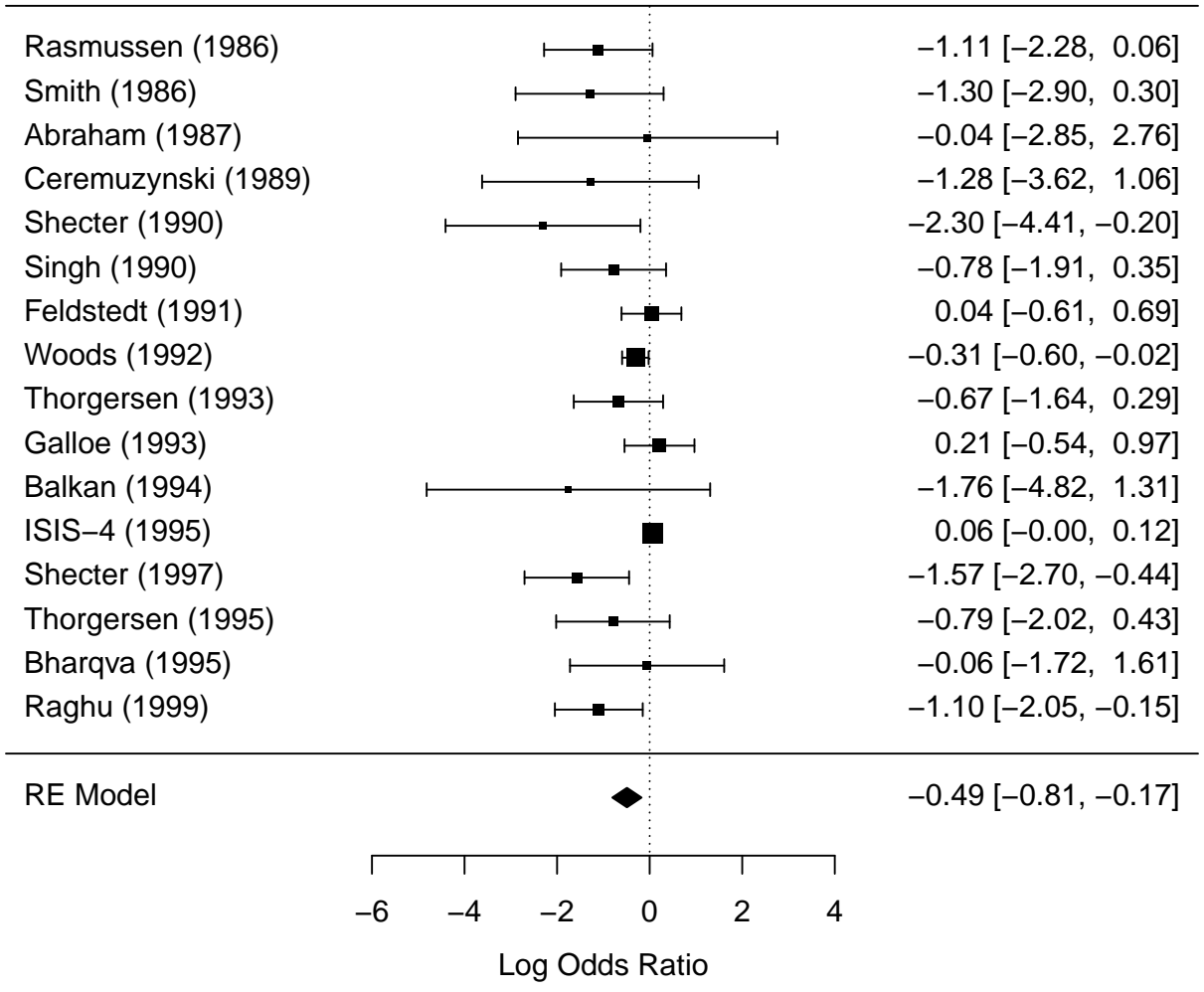

\section{Supplementary example: Meta-analyses with different estimated means, but similar evidence strength}

Here, we extend Example 3 to illustrate comparison between meta-analyses in which estimated means differ somewhat, yet due to differences in heterogeneity, the proposed metrics suggest comparable proportions of scientifically meaningful effect sizes. Others have conducted a series of cumulative meta-analyses of the literature on intravenous magnesium on mortality following acute myocardial infarction ${ }^{17}$. In a metaanalysis of the earliest 16 trials versus that of all 19 trials published at the time of reference 17's analysis, the estimated mean shifted from an odds ratio $(O R)$ of 0.61 (95\% CI: $0.45,0.84)$ to 0.72 (95\% CI: 0.58, 0.9). Considering inverse associations below $O R=0.8$ to be scientifically important, we reanalyzed data ${ }^{17}$ to estimate that, in the population of effects represented by the first 16 studies, approximately $76 \%$ are below $O R=0.8$ (95\% CI: 41\%, 100\%) versus approximately $66 \%$ (95\% CI: $28 \%, 100 \%)$ in the effects represented by all 19 studies (Supplementary Figure 1). Considering effects in the opposite direction, the two meta-analyses 
would estimate, respectively, that approximately $4 \%$ (95\% CI: 0\%, 6\%) and $2 \%(95 \%$ CI: $0 \%, 7 \%)$ are above an odds ratio of 1.2 .

Supplementary Figure 6: Estimated proportion of odds ratios (shaded) more protective than threshold of scientific importance at $O R=0.8$ (solid red line) in cumulative meta-analyses of 16 (gray) or 19 (orange) studies. Dashed red line: reference null value $(O R=1.0)$. (As usual, normality was assumed on the log-OR scale; the plotted distributions are skewed due to exponentiation.)

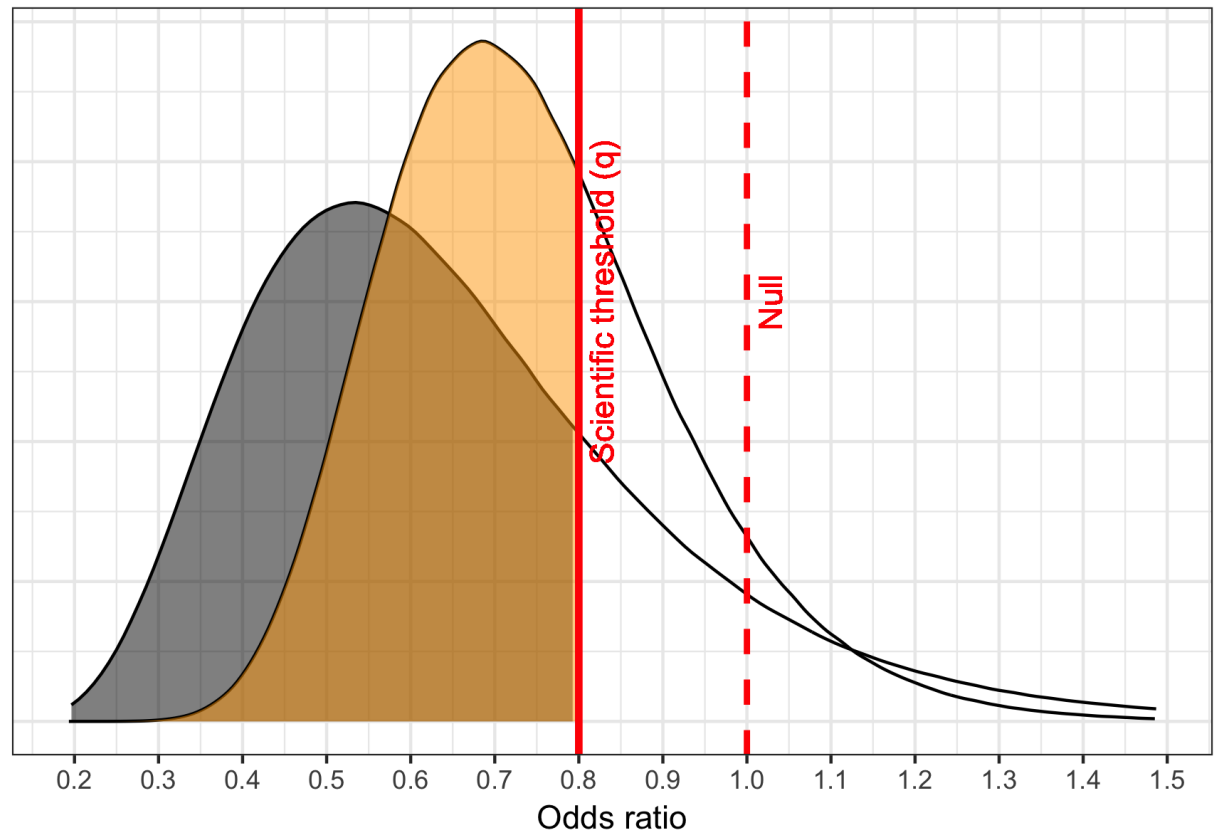

Under these thresholds of scientific importance, we might therefore consider both meta-analyses to have provided fairly strong evidence for scientifically important effects of magnesium again subject to methodological caveats mentioned in the main text ${ }^{18}$. This suggests a more stable view of evidence strength in an evolving literature than the difference in pooled point estimates alone might suggest.

\section{Simulation study}

We performed a simulation study assessing the relative coverage of the proposed asymptotic confidence interval (CI) versus a bootstrapped confidence interval, including in meta-analyses of few studies or with relatively low-powered studies. We fixed the mean of the true effects to $\mu=0.50$ on the mean difference scale while varying the number of studies $(k)$ between 10 and 50 , the heterogeneity $\left(\tau^{2} \in\{0.01,0.04,0.25\}\right)$, and the mean sample size in each study $(E[N] \in\{150,850\})$. We used a bias-corrected and accelerated bootstrap (BCa) with 10,000 iterates to estimate the bootstrap confidence intervals ${ }^{19}$. (Additional simulations, not shown, suggested that basic or percentile bootstrap methods yielded substantially worse performance than BCa.) On some simulation repetitions, the BCa method failed to converge with fewer iterates; we selected 10,000 iterates in order to ensure that every scenario had $<10 \%$ missing data due to convergence failures. Additional simulation results suggested that using fewer iterates (e.g., 5,000) yielded nearly identical confidence intervals, albeit with more frequent convergence failures in some scenarios. Therefore, in practice, we believe that 5,000 iterates is a reasonable choice unless the procedure fails to converge. 
Supplementary Figures 7 and 8, respectively, show coverage and width of theoretical vs. bootstrapped confidence intervals. With a true proportion $P \geq 0.15$, the theoretical confidence interval had approximately nominal coverage when $k=50$, regardless of the sample size of the meta-analyzed studies, and it usually had coverage $\geq 90 \%$ when $k \geq 10$. When $P \geq 0.15$, its overall mean coverage was $91 \%$, and its minimum coverage was $84 \%$. For a smaller true proportion $P=0.10$, the theoretical confidence interval sometimes showed fairly poor coverage for small $k$ (Supplementary Figure 7, Panel A). Across all scenarios, including $P=0.10$, the mean coverage of the theoretical interval remained $91 \%$, but its minimum coverage dropped slightly to $81 \%$. In contrast, across all scenarios, the BCa interval had nominal mean coverage $95 \%$ and minimum coverage $86 \%$. Accordingly, the bootstrap confidence interval were wider than the theoretical confidence interval, sometimes considerably so for small $k$ (Supplementary Figure 8). The bootstrap interval was sometimes overly conservative when the meta-analyzed studies were small (Supplementary Figure 8, Panel A, row 1). 
Supplementary Figure 7: Coverage of theoretical vs. bootstrapped 95\% confidence intervals. Dashed line indicates nominal coverage. True $\mathrm{P}=$ theoretical proportion of effects stronger than $q$ based on data generation parameters.
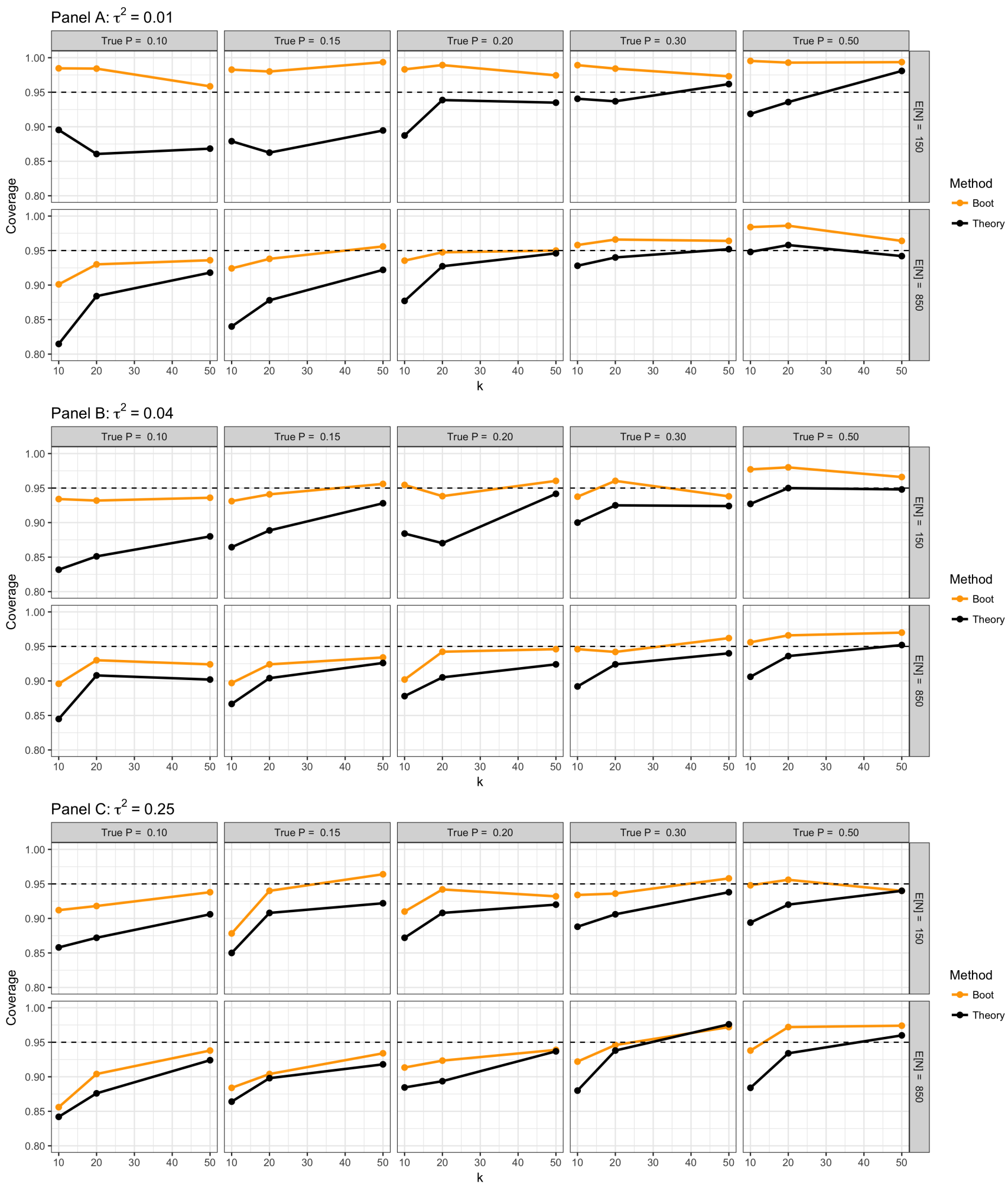

Method 
Figure 8: Width of theoretical vs. bootstrapped $95 \%$ confidence intervals. True $\mathrm{P}=$ theoretical proportion of effects stronger than $q$ based on data generation parameters.
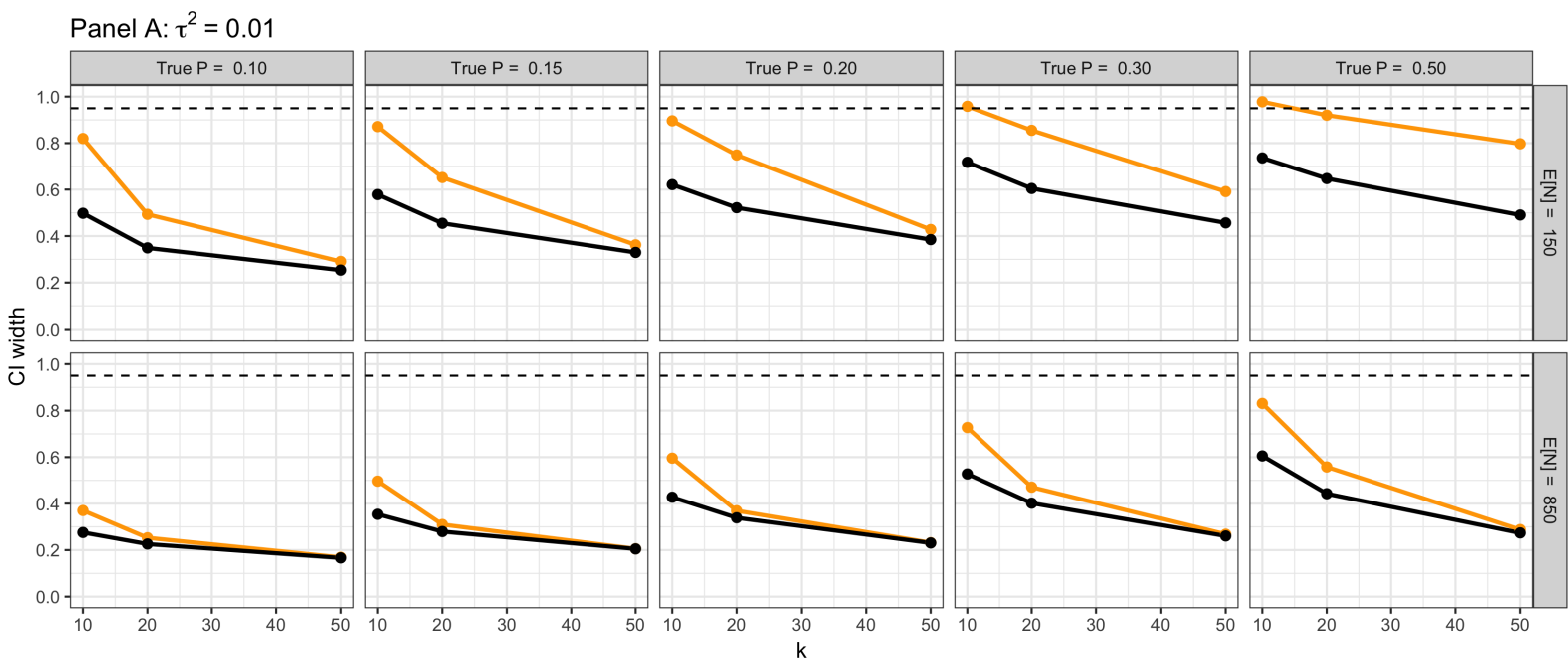

Method

$\rightarrow-$ Boot

$\rightarrow$ Theory
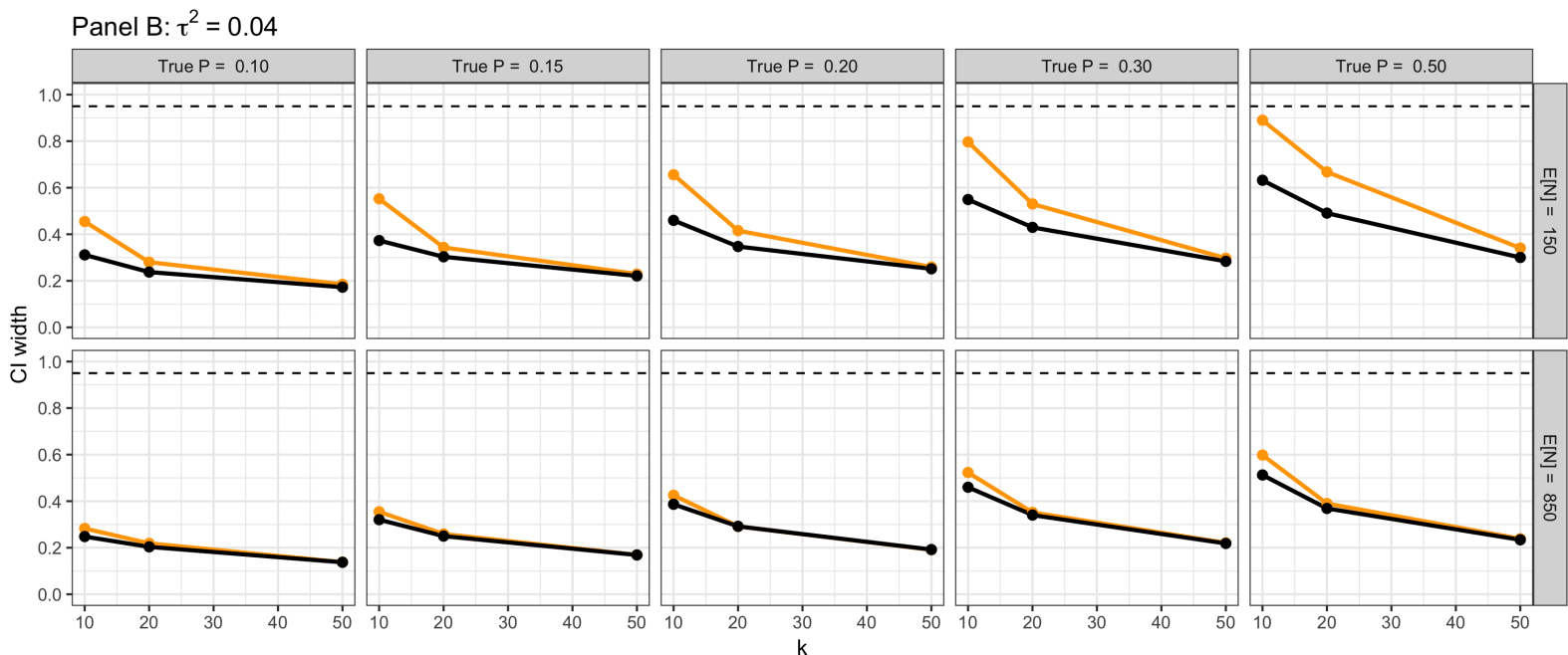

Method

$\rightarrow$ Boot

$\rightarrow$ Theory
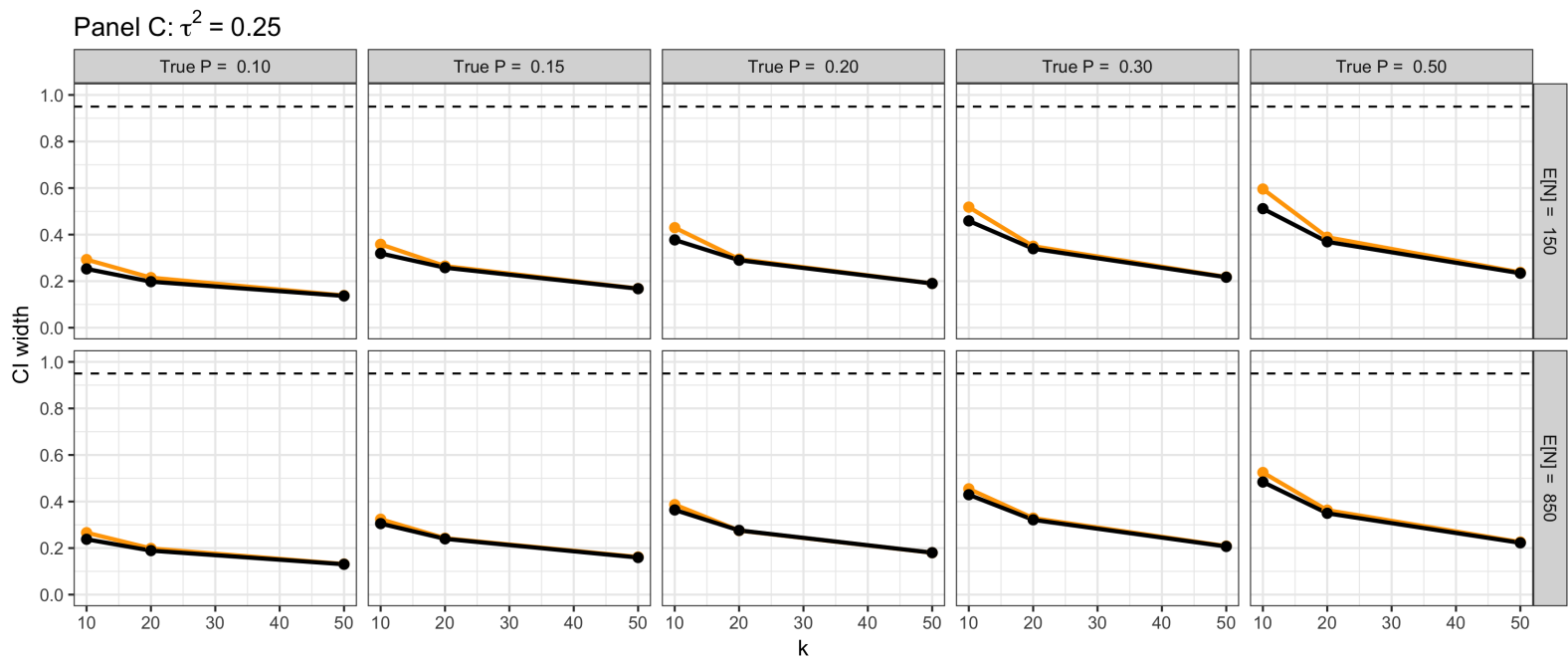

Method 


\section{References}

1. Brockwell SE, Gordon IR. A comparison of statistical methods for meta-analysis. Statistics in Medicine. 2001;20(6):825-840.

2. DerSimonian R, Laird N. Meta-analysis in clinical trials. Controlled Clinical Trials. 1986;7(3):177-188.

3. Paule RC, Mandel J. Consensus values and weighting factors. Journal of Research of the National Bureau of Standards. 1982;87(5):377-385.

4. Sidik K, Jonkman JN. Simple heterogeneity variance estimation for meta-analysis. Journal of the Royal Statistical Society: Series C (Applied Statistics). 2005;54(2):367-384.

5. Hedges L, Olkin I. Statistical Methods for Meta-Analysis. Academic Press; 1985.

6. Crosby RD, Kolotkin RL, Williams GR. Defining clinically meaningful change in health-related quality of life. Journal of clinical epidemiology. 2003;56(5):395-407.

7. Johnson PA, Goldman L, Orav EJ, Garcia T, Pearson SD, Lee TH. Comparison of the medical outcomes study short-form 36-item health survey in black patients and white patients with acute chest pain. Medical Care. 1995:145-160.

8. Hill CJ, Bloom HS, Black AR, Lipsey MW. Empirical benchmarks for interpreting effect sizes in research. Child Development Perspectives. 2008;2(3):172-177.

9. Müezzinler A, Zaineddin AK, Brenner H. A systematic review of leukocyte telomere length and age in adults. Ageing Research Reviews. 2013;12(2):509-519.

10. Braithwaite RS, Meltzer DO, King Jr JT, Leslie D, Roberts MS. What does the value of modern medicine say about the \$50,000 per quality-adjusted life-year decision rule? Medical Care. 2008;46(4):349-356.

11. Eichler H-G, Kong SX, Gerth WC, Mavros P, Jönsson B. Use of cost-effectiveness analysis in health-care resource allocation decision-making: How are cost-effectiveness thresholds expected to emerge? Value in Health. 2004;7(5):518-528.

12. Jaeschke R, Singer J, Guyatt GH. Measurement of health status: Ascertaining the minimal clinically important difference. Controlled Clinical Trials. 1989;10(4):407-415.

13. Redelmeier DA, Guyatt GH, Goldstein RS. Assessing the minimal important difference in symptoms: A comparison of two techniques. Journal of Clinical Epidemiology. 1996;49(11):1215-1219.

14. Norman GR, Sloan JA, Wyrwich KW. Interpretation of changes in health-related quality of life: The remarkable universality of half a standard deviation. Medical Care. 2003;41(5):582-592.

15. Chinn S. A simple method for converting an odds ratio to effect size for use in meta-analysis. Statistics in Medicine. 2000;19(22):3127-3131.

16. VanderWeele TJ. On a square-root transformation of the odds ratio for a common outcome. Epidemiology. 2017;28(6):e58-e60.

17. Shrier I, Boivin J, Platt R, et al. The interpretation of systematic reviews with meta-analyses: An objective 
or subjective process? BMC Medical Informatics and Decision-Making. 2008;8(1):19.

18. Higgins JP, Spiegelhalter DJ. Being sceptical about meta-analyses: A Bayesian perspective on magnesium trials in myocardial infarction. International Journal of Epidemiology. 2002;31(1):96-104.

19. Efron B. Better bootstrap confidence intervals. Journal of the American Statistical Association. 1987;82(397):171-185. 